\title{
Cosmological constant, gauge hierarchy, and warped geometry
}

\author{
Stephan J. Huber* \\ Deutsches Elektronen-Synchrotron DESY, Hamburg, Germany \\ Qaisar Shafi ${ }^{\dagger}$ \\ Bartol Research Institute, University of Delaware, Newark, Delaware 19716, USA
}

(Received 16 August 2002; published 1 July 2003)

\begin{abstract}
It is suggested that the mechanism responsible for the resolution of the gauge hierarchy problem within the warped geometry framework can be generalized to provide a new explanation of the extremely tiny vacuum energy density $\rho_{V}$ suggested by recent observations. We illustrate the mechanism with some 5D examples in which the true vacuum energy is assumed to vanish, and $\rho_{V}$ is associated with a false vacuum energy such that $\rho_{V}^{1 / 4} \sim \mathrm{TeV}^{2} / M_{\mathrm{Pl}} \sim 10^{-3} \mathrm{eV}$, where $M_{\mathrm{Pl}}$ denotes the reduced Planck mass. We also consider a quintessence-like solution to the dark energy problem.
\end{abstract}

DOI: 10.1103/PhysRevD.68.023503

PACS number(s): 98.80.Cq, 11.10.Kk

\section{INTRODUCTION}

Evidence for a tiny but non-zero cosmological vacuum energy density has steadily mounted in recent years [1]. With information from a variety of observations put together, it appears that we are living in a flat universe, such that $\Omega_{\Lambda}$ $+\Omega_{m}=1$, where $\Omega_{\Lambda}=0.7$ and $\Omega_{m}=0.3$ denote the density parameters associated with the vacuum energy and matter respectively. These observations pose at least two formidable theoretical challenges: namely, what physics determines the magnitude of the vacuum energy density to be of order

$$
\rho_{V} \sim 10^{-120} M_{\mathrm{Pl}}^{4} \sim\left(10^{-3} \mathrm{eV}\right)^{4},
$$

and why are $\rho_{V}$ and $\rho_{m}$ in such close proximity in magnitude, considering that their evolution, at least for constant cosmological constant, can be so different? Here $M_{\mathrm{Pl}}=2.4$ $\times 10^{18} \mathrm{GeV}$ denotes the reduced Planck mass.

A certain amount of theoretical prejudice suggests that the true vacuum energy density of the universe could be exactly zero. Needless to say, how the zero value occurs remains a complete mystery, despite many attempts [2]. Nonetheless, following this reasoning, it has been speculated that the present universe happens to lie in a false vacuum, separated from the true vacuum by the observed energy density $\rho_{V}[3]$. The modest goal of this approach is to try to identify the physics underlying the origin of $\rho_{V}$, as well as ensure that the metastable vacuum is sufficiently long lived.

In this paper we propose a new mechanism for realizing $\rho_{V}$ of the desired magnitude which is based on warped geometry. While the original motivation of warped geometry was to resolve the notorious gauge hierarchy problem $[4,5]$, it has subsequently been exploited to provide at least a qualitatively new understanding of fermion mass hierarchies and their mixings, especially within the context of neutrino oscillations [6-9]. We will show here that the warped geometry can be further exploited to provide a new mechanism for

\footnotetext{
*Email address: stephan.huber@desy.de

†Email address: shafi@bartol.udel.edu
}

generating $\rho_{V}$ of the desired magnitude. Earlier attempts in this direction have been made in Ref. [10]. Recall that the $\mathrm{TeV}$ scale in warped geometry arises from the presence of the warp factor $\Omega^{-1}=\exp (-\pi k R)=\mathrm{TeV} / M_{P}$, where $1 / k$ denotes the AdS curvature radius and $R$ is the radius of the orbifold $S^{1} / Z_{2}$. We will see that $\rho_{V}$ can be associated with the vacuum energy density of a suitable scalar field, such that

$$
\rho_{V}^{1 / 4} \sim \Omega^{-2} M_{\mathrm{Pl}}=\mathrm{TeV}^{2} / M_{\mathrm{Pl}} \sim 10^{-3} \mathrm{eV} .
$$

We also discuss how the quintessence scenario [11] can be realized within this approach.

\section{SCALAR FIELDS IN A WARPED BACKGROUND}

We take the fifth dimension to be an $S_{1} / Z_{2}$ orbifold with a negative bulk cosmological constant, bordered by two 3 -branes with opposite tensions and separated by distance $R$. Einstein's equations are satisfied by the non-factorizable metric [4]

$$
d s^{2}=e^{-2 \sigma(y)} \eta_{\mu \nu} d x^{\mu} d x^{\nu}+d y^{2}, \quad \sigma(y)=k|y|,
$$

which describes a slice of $\mathrm{AdS}_{5}$. The 4-dimensional metric is $\eta_{\mu \nu}=\operatorname{diag}(-1,1,1,1), k$ is the AdS curvature related to the bulk cosmological constant and brane tensions, and $y$ denotes the fifth coordinate. The AdS curvature and 5D Planck mass $M_{5}$ are both assumed to be of order $M_{\mathrm{Pl}}$. The AdS warp factor $\Omega=e^{\pi k y}$ generates an exponential hierarchy of energy scales. If the brane separation is $k R \simeq 11$, the scale at the negative tension brane, located at $y=\pi R$, is of $\mathrm{TeV}$ size, while the scale at the brane at $y=0$ is of order $M_{\mathrm{Pl}}$. At the $\mathrm{TeV}$-brane gravity is weak because the zero mode corresponding to the $4 \mathrm{D}$ graviton is localized at the positive tension brane (Planck-brane).

Let us consider the equation of motion of a real scalar field in the background (2) $[12,7]$

$$
\frac{1}{\sqrt{-g}} \partial_{M}\left(\sqrt{-g} g^{M N} \partial_{N} \Phi\right)-M^{2} \Phi=0
$$

which follows from the action 


$$
S_{5}=-\int d^{4} x \int d y \sqrt{-g}\left(\frac{1}{2}\left(\partial_{M} \Phi\right)\left(\partial^{M} \Phi\right)+\frac{1}{2} M^{2} \Phi^{2}\right) .
$$

Here $g_{M N}$ denotes the 5D metric and $g=e^{-4 \sigma}$ its determinant. In general, the 5D scalar mass consists of bulk and brane contributions

$$
M^{2}(y)=b^{2} k^{2}+a^{2} k \delta(y-\pi R)+\tilde{a}^{2} k \delta(y),
$$

where $b, a$ and $\tilde{a}$ are dimensionless parameters. In the case of supersymmetry the relations

$$
\begin{aligned}
& \tilde{a}^{2}=-a^{2} \\
& b^{2}=\frac{1}{4} a^{2}\left(a^{2}+8\right)
\end{aligned}
$$

have to be satisfied [7], and $\Phi$ becomes a complex scalar field which, together with a second complex scalar and a Dirac fermion, forms a hypermultiplet. The connection to the 5D mass parameter of the Dirac fermion, $M_{\Psi}=c \sigma^{\prime}$, is given by $c=3 / 2+a^{2} / 2$. A special case of Eqs. (6) is the $m$ assless scalar field where $a^{2}=\tilde{a}^{2}=b=0$ (and $c=3 / 2$ ).

The effective 4D theory arises from the Kaluza-Klein (KK) decomposition

$$
\Phi\left(x^{\mu}, y\right)=\frac{1}{\sqrt{2 \pi R}} \sum_{n=0}^{\infty} \Phi^{(n)}\left(x^{\mu}\right) f_{n}(y),
$$

where the wave functions $f_{n}(y)$ satisfy the differential equation $[12,7]$

$$
\left(-\partial_{5}^{2}+4 \sigma^{\prime} \partial_{5}+M^{2}\right) f_{n}=e^{2 \sigma} m_{n}^{2} f_{n},
$$

and $m_{n}$ are the masses of the KK excitations. The solution to Eq. (8) is given by

$$
f_{n}(y)=\frac{e^{2 \sigma}}{N_{n}}\left[J_{\alpha}\left(\frac{m_{n}}{k} e^{\sigma}\right)+\beta_{\alpha}\left(m_{n}\right) Y_{\alpha}\left(\frac{m_{n}}{k} e^{\sigma}\right)\right],
$$

with $\alpha=\sqrt{4+b^{2}}$. Assuming $\Phi$ is even under the $Z_{2}$ orbifold transformation, i.e. $f_{n}(-y)=f_{n}(y)$, the coefficient $\beta$ and the $\mathrm{KK}$ spectrum follow from the matching conditions at the branes,

$$
\begin{aligned}
& \beta_{\alpha}\left(x_{n}, \tilde{a}^{2}\right)=-\frac{\left(-\tilde{a}^{2}+4-2 \alpha\right) J_{\alpha}\left(x_{n}\right)+2 x_{n} J_{\alpha-1}\left(x_{n}\right)}{\left(-\tilde{a}^{2}+4-2 \alpha\right) Y_{\alpha}\left(x_{n}\right)+2 x_{n} Y_{\alpha-1}\left(x_{n}\right)}, \\
& \beta_{\alpha}\left(x_{n}, \tilde{a}^{2}\right)=\beta_{\alpha}\left(\Omega x_{n},-a^{2}\right),
\end{aligned}
$$

where $x_{n}=m_{n} \Omega / k$. Note that for non-vanishing boundary mass terms the derivative of $f_{n}$ becomes discontinuous on the boundaries. The normalization constants $N_{n}$ in Eq. (9) are defined such that

$$
\frac{1}{2 \pi R} \int_{-\pi R}^{\pi R} d y e^{-2 \sigma(y)} f_{n}^{2}(y)=1 .
$$

The mass splitting between the $\mathrm{KK}$ modes is $\Delta m_{n}$ $\sim \pi k \Omega^{-1} \sim \mathrm{TeV}$.

In the massless case the zero mode wave function is constant, i.e. $f_{0}(y)=1 / N_{0} \approx \sqrt{2 \pi k R}$. The KK states have $\mathrm{TeV}$ scale masses and are localized at the TeV-brane. The mass of the first excited state is $m_{1} \approx 3.83 k \Omega^{-1}$. The effects of the bulk and brane mass terms on the mass and wave function of the lowest mode are quite different. If one switches on a small bulk mass, one finds $m_{0} \approx b k / \sqrt{2}$ for $b \leqq \Omega^{-1}$ ( $b k$ $\lesssim \mathrm{TeV}$ ). Thus, there is no warp factor suppression. The zero mode wave function is still approximately constant, with a small enhancement at the TeV-brane. Increasing the bulk mass to $b \leq 1$ ( $\left.b k \leq M_{\mathrm{Pl}}\right)$ the mass of the lightest mode remains frozen at $x_{0}=3.83$, which is practically the former first excited state. For $b \gtrsim 1\left(b k \geq M_{\mathrm{Pl}}\right)$ one finds that $m_{0}$ also increases. The mass term at the Planck-brane induces a similar behavior of the zero mode, except there is no increase of $m_{0}$ for $\tilde{a} \gtrsim 1\left(\tilde{a} k \gtrsim M_{\mathrm{Pl}}\right)$.

In the case of a $\mathrm{TeV}$-brane mass the result is very different. Because of the factor $\sqrt{-g}$ in the action (4), the mass of the zero mode is warped by two powers of $\Omega$. With this double warping we have

$$
m_{0} \approx a k \Omega^{-2}, \quad a \lesssim 1 .
$$

The wave function is nearly constant, with a dip at the TeVbrane, as shown in Fig. 1(a) for $k R=11.13\left(M_{\mathrm{Pl}} e^{-\pi k R}\right.$ $=1.6 \mathrm{TeV})$. For $a \gtrsim 1$ the dip in the wave function slows down the increase of $m_{0}$. For large values of $a$ any further increase is completely compensated by this, so that the zero mode mass saturates at $m_{0}=2 \sqrt{2} k \Omega^{-2}$. The dependence of $m_{0}$ on the TeV-brane mass is displayed in Fig. 1(b). We note that gauge bosons show a similar behavior as we discussed in Ref. [13]. Numerically, for a TeV-brane mass of order $M_{\mathrm{Pl}}, m_{0}$ is comparable to the energy scale associated with dark energy (1). Of course, at this stage $m_{0}$ is not yet related to some vacuum energy, but merely represents a tiny particle mass. In the next section we will show how such a link can be established.

At the end of this section, let us briefly summarize the supersymmetric case which was extensively discussed in Refs. $[7,14]$. Supersymmetry requires the bulk and brane mass terms to be simultaneously present. Because of the special relations, Eqs. (6), the zero mode remains strictly massless, despite the non-zero bulk and brane masses. The wave function has an exponential form [7]

$$
f_{0}(y)=\frac{1}{N_{0}} e^{-\left(a^{2} / 2\right) \sigma} .
$$

For a positive (negative) mass squared term at the TeV-brane the scalar field is localized towards the Planck-brane (TeVbrane). The supersymmetric scalar fields thus behave similar to fermions in the warped background, which also possess massless exponential zero modes in the presence of bulk mass terms $[6,7]$. 

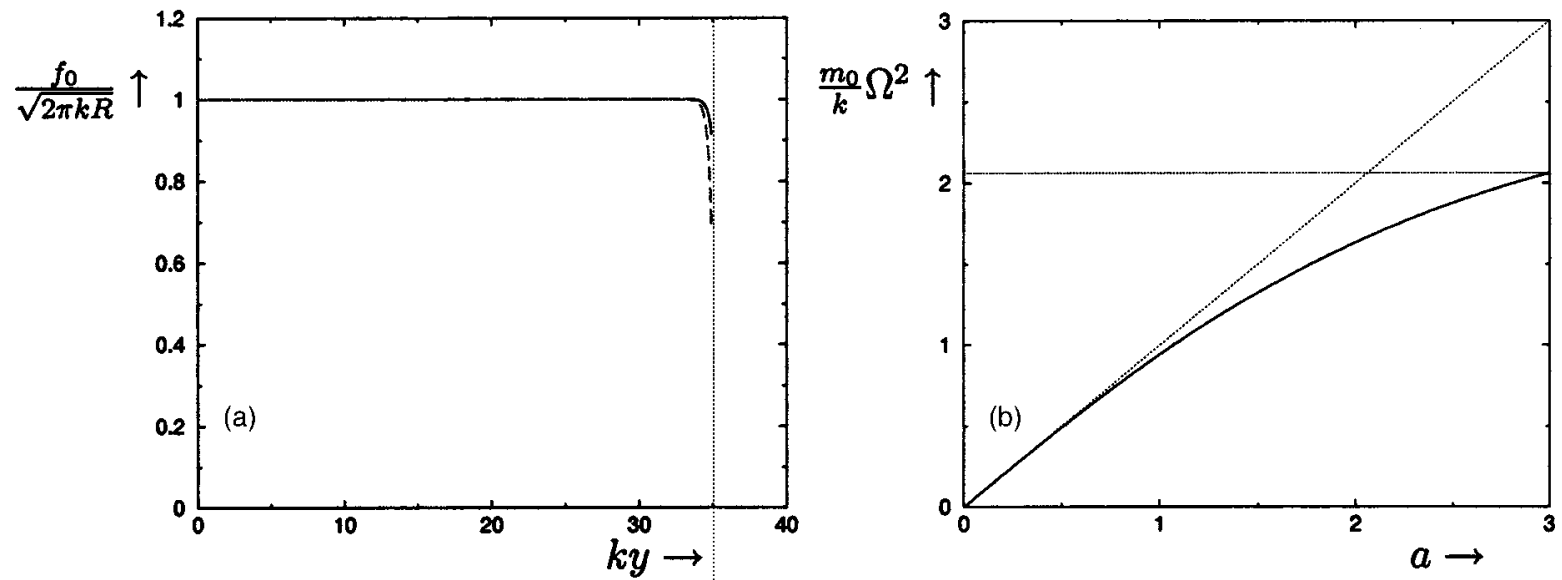

FIG. 1. (a) Wave function of the zero mode for $a=1$ (solid line) and $a=2$ (dashed line). (b) Dependence of the zero mode mass on the TeV-brane mass term (solid line) compared to the linear approximation of Eq. (13) (dotted line).

\section{EXPONENTIALLY SMALL CONTRIBUTIONS TO THE SCALAR POTENTIAL}

In the last section we learned that a Planck-size mass term at the TeV-brane is translated into a sub-eV mass of the scalar particle. We now generalize this result to include a scalar potential on the TeV-brane. This will allow us to generate tiny energy splittings in the minima of the 4D effective potential, which we hope to identify with the observed dark energy.

We start with the simplest case of a massless scalar field with $a=b=\tilde{a}=0$ which, for instance, could be a consequence of supersymmetry. If we now switch on some general potential on the TeV-brane, related, for example, to supersymmetry breaking, the scalar zero mode remains, as discussed in the last section. Consider the following contribution to the 5D action:

$$
\begin{aligned}
\Delta S_{5}= & -\int d^{4} x \int d y \sqrt{-g}\left(\frac{1}{2} a^{2} k \Phi^{2}+\frac{1}{3} E_{5} k^{-1 / 2} \Phi^{3}\right. \\
& \left.+\frac{1}{4} \lambda_{5} k^{-2} \Phi^{4}\right) \delta(y-\pi R) .
\end{aligned}
$$

We approximate $f_{0}$ by a constant, and after integrating over the extra dimension, we arrive at the 4D effective potential for the zero mode $\phi$ :

$$
V_{4}=\frac{1}{2} m^{2} \phi^{2}+\frac{1}{3} E \phi^{3}+\frac{1}{4} \lambda \phi^{4},
$$

where

$$
\begin{gathered}
m^{2}=a^{2} k^{2} \Omega^{-4} \\
E=E_{5} k \Omega^{-4} \\
\lambda=\lambda_{5} \Omega^{-4} .
\end{gathered}
$$

Thus all operators on the TeV-brane are redshifted by four powers of the warp factor. The warped geometry not only generates tiny masses but also exponentially small coupling constants. This result may seem surprising since the zero mode $f_{0}$ is constant in the extra dimension and thus has a large overlap with the TeV-brane. However, as we observe from Eq. (12), in the integration over the extra dimension the region close to the $\mathrm{TeV}$-brane is exponentially suppressed. The rescaled zero mode $\widetilde{f}_{0}=e^{-\sigma} f_{0}$ is the more appropriate object to look at. For a constant $f_{0}$, the rescaled zero mode is exponentially localized towards the Planck-brane, which explains the tiny quantities in Eq. (17). For a very large mass squared term on the TeV-brane, i.e. $a \gg 1$, there is an additional suppression of the brane operators (15) induced by the dip in the zero mode. We note that scalar couplings in the bulk or on the Planck-brane are not scaled down by the warped geometry.

We now assume that $m^{2}<0$ in order to generate a vacuum expectation value (VEV) for the scalar field. Without additional interactions from the bulk, the tiny self-coupling $\lambda$ induced by the brane can stabilize the field only at a Plancksize $\operatorname{VEV}\langle\phi\rangle \sim k$. We therefore have to introduce some additional interaction. A quartic coupling in the bulk does not feel the warping and therefore generates $\lambda \sim 1$ in the effective 4D action. A quartic coupling on the Planck-brane would have the same effect. The scalar field then acquires a tiny $\operatorname{VEV}\langle\phi\rangle \sim k \Omega^{-2}$. If there is now more than one minimum with a similar VEV, the energy splitting between the two minima will be on the order of $\Delta V \sim m^{2}\langle\phi\rangle^{2} \sim k^{4} \Omega^{-8}$. This is exactly what is required for the dark energy. Two minima can easily arise, for example, in the case of two interacting scalar fields with a polynomial potential. We will show later that the false vacuum has a lifetime much longer than the age of the universe. In the next section we will also discuss how this setup can be stabilized against radiative corrections in a supersymmetric framework.

A different possibility for the scalar field to be stabilized is by some non-renormalizable interaction in the bulk, for instance by a $\left(1 / Q^{2}\right) \phi^{6}$ operator. As discussed in Refs. [7,8], non-renormalizable interactions may be suppressed by much smaller energy scales than $M_{\mathrm{Pl}}$ because of the warped geom- 
etry. However, as explained above, in some sense the flat zero mode is localized towards the Planck-brane. Therefore non-renormalizable operators are not enhanced, and we expect $Q \sim M_{\mathrm{Pl}}=k$ in the $4 \mathrm{D}$ action. The scalar would then be stabilized at $\langle\phi\rangle=\sqrt{m Q} \sim k \Omega^{-1}$, which is the KK scale. Assuming that the $Z_{2}$ symmetry in the scalar potential is broken by the cubic term on the brane, the induced energy splitting between the vacua at $\pm \sqrt{m Q}$ is $\Delta V \sim E\langle\phi\rangle^{3} \sim k^{4} \Omega^{-7}$. This is a factor of $\Omega$ larger than what we found in the previous example. To make it compatible with the observational value (1) we either would have to increase the warp factor and end up with a KK scale of about $10 \mathrm{GeV}$ (!) or reduce the suppression scale to about $Q=k \Omega^{-1 / 3}$. Both possibilities do not look realistic. In the next section we discuss the supersymmetric case (with a non-vanishing bulk mass) which gives us additional possibilities.

The false vacuum energy not only leads to an exponential expansion of the universe, but will also modify the geometry of the extra dimension. To estimate the size of the back reaction we compare the false vacuum energy density to the bulk cosmological constant and brane tensions of the unperturbed Randall-Sundrum solution [4]. We consider a somewhat simplified setup where the scalar VEV is induced by a negative squared mass term $(1 / 2) a^{2} k \Phi^{2}$ at the TeV-brane, as in Eq. (15). The potential is stabilized by a quartic selfinteraction in the bulk, $(1 / 4) \lambda_{b} \Phi^{4}$. Because the scalar potential changes along the extra dimension, the vacuum configuration $\langle\Phi(y)\rangle$ does as well and has to be computed numerically. We find the profile to be $\langle\Phi(y)\rangle$ $\sim a k \Omega^{-2} / \sqrt{\lambda_{\mathrm{b}}} \sim k^{3 / 2} \Omega^{-2}$ and almost flat in the extra dimension. In the second step we assumed $a \sim 1$ and $\lambda_{\mathrm{b}} \sim k^{-1}$. Close to the TeV-brane, where the instability occurs, the profile $\langle\Phi(y)\rangle$ increases by about $10 \%$ relative to its almost constant value in the bulk. On the TeV-brane the scalar potential is $V=(1 / 2) a^{2} k \Phi^{2} \sim k^{4} \Omega^{-4}$. In the bulk we obtain $V$ $=(1 / 4) \lambda_{\mathrm{b}} \Phi^{4} \sim k^{5} \Omega^{-8}$. This means that Einstein's equations are still completely dominated by the Planck-size bulk cosmological constant and brane tensions of the RandallSundrum solution [4]. Thus we can safely ignore the back reaction, at most of order $\Omega^{-4}$, on the wave functions (9) and (14).

\section{SUPERSYMMETRIC CASE}

A crucial point is that a perturbation of the mass relations (6) by some supersymmetry breaking mass term on the TeVbrane with $\Delta a^{2} \leq 1$ leaves the zero mode (14) practically unchanged. For $\Delta a^{2} \geq 1$ we observe an additional reduction of $f_{0}$ close to the TeV-brane, as in the case of a vanishing bulk mass. To obtain the 4D couplings from the brane action (15) we approximate the zero mode by its supersymmetric shape (14). We find

$$
\begin{aligned}
m^{2} & \approx \Delta a^{2} k^{2} \Omega^{-4-a^{2}} \\
E & \approx E_{5} k \Omega^{-4-(3 / 2) a^{2}} \\
\lambda & \approx \lambda_{5} \Omega^{-4-2 a^{2}},
\end{aligned}
$$

where $a^{2}$ is now the TeV-brane mass term required by supersymmetry and determines the shape of the zero mode. We have omitted a factor $N_{0} \sqrt{2 \pi k R}$ to some power which is of order unity. Localizing the scalar zero mode towards the Planck-brane by taking $a^{2}>0$, we can suppress the induced 4D couplings even stronger than in Eq. (17). This opens up new possibilities to generate a small vacuum energy density.

The $(N=2) \quad 5 \mathrm{D}$ supersymmetry prohibits a selfinteraction of the scalar field in the bulk. Self-interactions can be provided, however, by superpotentials on the branes, where the boundary conditions break the supersymmetry to $N=1$. Planck-brane operators are not warped down and induce order unity couplings in the $4 \mathrm{D}$ potential, in contrast to their TeV-brane counterparts (18).

Localizing the scalar field close to the Planck-brane, we can implement a quintessence-like solution to the dark energy problem. The cosmological evolution is assumed to leave the scalar field displaced from its minimum towards which it is slowly evolving. For polynomial potentials, the necessary slow rolling conditions are satisfied only for very large VEVs $\langle\phi\rangle \sim M_{\mathrm{Pl}}$. A small vacuum energy can therefore be explained only by extraordinarily small couplings. To generate $\rho_{V}$ from a term $m^{2}\langle\phi\rangle^{2} \sim m^{2} M_{\mathrm{Pl}}^{2}$ requires $m^{2}$ $\sim \Omega^{-8} M_{\mathrm{Pl}}^{2}$. From Eq. (18) we see that this requires $a^{2} \approx 4$. We can also implement this scenario by relying on the quartic term of the brane potential, $\rho_{v} \sim \lambda\langle\phi\rangle^{4}$. For $a^{2} \sim 2$ we obtain the required small $\lambda \approx \Omega^{-8}$. The other terms in Eq. (15) as well as possible bulk self-interactions have to vanish to accommodate this solution. It remains to be seen whether a realistic scenario of this type can be constructed in which the required small masses remain stable under quantum corrections [15].

A true cosmological constant, instead of quintessence, can be generated from a supersymmetry breaking cubic coupling which shifts previously degenerate minima. We assume that a scalar self-interaction on the Planck-brane leads to $\lambda \sim 1$ in the $4 \mathrm{D}$ action. If the scalar potential is now destabilized by a supersymmetry breaking brane mass (18), the induced VEV is $\langle\phi\rangle \sim m \sim k \Omega^{-2-a^{2} / 2}$. The degeneracy of the minima at $\pm\langle\phi\rangle$ is lifted by the supersymmetry breaking cubic term on the brane (18). The induced energy splitting between the vacua is then of order $\Delta V \sim E\langle\phi\rangle^{3} \sim k^{4} \Omega^{-10-3 a^{2}}$. The preferred value of the cosmological constant (1) then leads to $a^{2} \sim-2 / 3$; i.e., the scalar field is localized somewhat towards the TeV-brane. The mass of the scalar particles associated with $\Phi$ is of order $\langle\phi\rangle \sim k \Omega^{-5 / 3} \sim 10^{2} \mathrm{eV}$.

\section{QUANTUM CORRECTIONS}

The case with a vanishing bulk mass discussed in Sec. III can readily be embedded in the supersymmetric framework by setting $a=0$ in Eq. (18). Then supersymmetry helps to tame radiative corrections. As in four dimensions the soft mass of the scalar zero mode, $\mathrm{m}^{2}$, will receive a 1-loop quantum correction $\delta m^{2} \sim\left(1 / 16 \pi^{2}\right) m^{2} \lambda \ln (\Lambda)$ by exchange of the scalar zero mode and its superpartner. Here $\Lambda$ denotes the momentum cutoff and $\lambda$ stems from the self-coupling localized at the Planck-brane. As discussed above, in the case 
$a=0$ we have $m^{2} \sim k^{2} \Omega^{-4}$ and $\lambda \sim 1$. In the warped model there are additional radiative corrections to $m^{2}$ by the exchange of KK states of the scalar field. The KK states are localized towards the TeV-brane. Therefore they acquire a larger soft mass of order $k^{2} \Omega^{-2}$. The quartic coupling between two zero modes and two KK states of the scalar field is of order $\Omega^{-2}$. Thus loops with KK states of the scalar are of the same order as zero mode loops. It remains to be seen if performing the sum over the KK contributions, which are individually small, leads to a destabilization of the soft mass of the zero mode.

Since we assume the scalar field to be a gauge singlet, further quantum corrections can only come from gravity. Exchange of a zero mode gravitino in the loop gives rise to $\delta m^{2} \sim\left(1 / 16 \pi^{2}\right) m_{3 / 2}^{2}\left(\Lambda / M_{\mathrm{Pl}}\right)^{2}$, where $m_{3 / 2}$ is the gravitino mass. Since for the zero mode of the gravitino $m_{3 / 2}$ $\sim k \Omega^{-3}$, we obtain a tiny correction even for a Planck-size cutoff. For the KK gravitinos the situation is different. Being localized towards the TeV-brane, their supersymmetry breaking mass is $\mathrm{TeV}$ size $\left(k \Omega^{-1}\right)$ [14] and their dimension-6 interaction with the scalar zero mode is suppressed by $1 /\left(\Omega M_{\mathrm{Pl}}^{2}\right)$. The corresponding correction to the scalar mass is then $\delta m^{2} \sim\left(1 / 16 \pi^{2}\right) k^{2} \Omega^{-3}\left(\Lambda / M_{\mathrm{Pl}}\right)^{2}$. Since the dimension-6 operator has its support at the TeV-brane, it seems plausible that the KK gravitino loop is cut off at the $\mathrm{TeV}$ scale. In this case its contribution to the scalar mass $\delta m^{2} \sim\left(1 / 16 \pi^{2}\right) k^{2} \Omega^{-5}$ is safely suppressed. However, given the limitations and uncertainties which are inherent in our treatment of radiative corrections, the issue of the quantum stability of our approach to the dark energy problem is not yet satisfactorily settled.

\section{LIFETIME OF THE FALSE VACUUM}

The false vacuum state is metastable and will finally relax to the true vacuum by thermal fluctuations and/or quantum tunneling. We must require the lifetime of the false vacuum to be longer than the age of the universe. Because of the low temperature of the present universe, quantum tunneling is the dominant decay process. In the WKB approximation the tunneling probability is given by [16]

$$
p \sim\left(\frac{t_{U}}{R}\right)^{4} e^{-B}
$$

where $t_{U}$ denotes the age of the universe, and $R \sim 1 / m$ is the characteristic scale of the problem. In the thin wall approximation the Euclidean action of the tunneling configuration,

$$
B=27 \pi^{2} \frac{S_{1}^{4}}{2(\Delta V)^{3}},
$$

depends on the energy splitting of the vacua $\Delta V$ and the "surface tension" of the bubble $S_{1}=\int d \phi \sqrt{2 V(\phi)}$.

Vacuum decay is most effective between minima which are close to each other in field space and separated only by a low energy barrier $V_{B}$. Among the examples discussed before the tunneling rate is largest in the case of Sec. III where $m \sim\langle\phi\rangle \sim 10^{-3} \mathrm{eV}$. From the constraint $p<1$ we obtain $B$ $\gtrsim 280$. For a polynomial potential one finds $B \approx 7$ $\times 10^{3}\left(V_{B} / \Delta V\right)^{3} / \lambda$; i.e., the false vacuum is metastable even for $V_{B} \sim \Delta V$, as we expect for a potential with only a single mass scale. In the other example we discussed, the barrier heights and scalar VEVs are even much larger. Thus, in all the cases discussed the false vacuum is sufficiently long lived.

\section{CONCLUSIONS}

In conclusion, we have shown that a warped geometry setting could help provide a new explanation of the origin of the tiny vacuum energy density of the universe that is indicated by recent observations. This can either be in the form of a cosmological constant or, if supersymmetry is invoked, as a contribution arising from a slow rolling quintessence field. Although no explanation exists as to why all other contributions to the vacuum energy density effectively vanish, it is certainly intriguing to think that resolution of the gauge hierarchy and dark energy problems may have a common origin.

\section{ACKNOWLEDGMENTS}

We acknowledge useful discussions with A. Hebecker and A. Pomarol and a helpful correspondence with T. Gherghetta. We wish to thank the Alexander von Humboldt Stiftung for providing the impetus for our collaboration. Q.S. also acknowledges the hospitality of the Theory Groups at DESY and Heidelberg, especially Wilfried Buchmüller, Michael Schmidt and Christof Wetterich. This work was supported in part by the DOE under contract DE-FG02-91ER40626.
[1] S. Perlmutter et al., Astrophys. J. 483, 565 (1997); S. Perlmutter et al., Bull. Am. Astron. Soc. 29, 1351 (1997); B. Schmidt et al., Astrophys. J. 507, 46 (1998); A.J. Riess et al., Astron. J. 116, 1009 (1998); Astrophys. J. 560, 49 (2001).

[2] For recent reviews of various suggestions for resolving the cosmological constant problems and additional references, see A. Vilenkin, hep-th/0106083; U. Ellwanger, hep-ph/0203252.

[3] S. Kachru, J. Kumar, and E. Silverstein, Phys. Rev. D 59, 106004 (1999); E.I. Guendelman, Mod. Phys. Lett. A 14, 1043 (1999); P.H. Frampton, hep-th/0002053; N. Arkani-Hamed, L.J. Hall, C. Colda, and H. Murayama, Phys. Rev. Lett. 85, 4434 (2000); S.M. Barr and D. Seckel, Phys. Rev. D 64,
123513 (2001); T. Banks and M. Dine, J. High Energy Phys. 10, 012 (2001).

[4] L. Randall and R. Sundrum, Phys. Rev. Lett. 83, 3370 (1999).

[5] M. Gogberashvili, Int. J. Mod. Phys. D 11, 1635 (2002).

[6] Y. Grossman and M. Neubert, Phys. Lett. B 474, 361 (2000).

[7] T. Gherghetta and A. Pomarol, Nucl. Phys. B586, 141 (2000).

[8] S.J. Huber and Q. Shafi, Phys. Lett. B 498, 256 (2001).

[9] S.J. Huber and Q. Shafi, Phys. Lett. B 521, 365 (2001); ibid. 544, 295 (2002).

[10] S.H. Henry Tye and I. Wasserman Phys. Rev. Lett. 86, 1682 (2001); J.M. Cline and H. Firouzjahi, Phys. Lett. B 514, 205 (2001). 
[11] C. Wetterich, Nucl. Phys. B302, 668 (1988); P.J.E. Peebles and B. Ratra, Astrophys. J., Lett. Ed. 325, L17 (1988); R. R Caldwell, R. Dave, and P. J Steinhard, Phys. Rev. Lett. 80, 1582 (1998); I. Zlatev, L. Wang, and P. J Steinhard, ibid. 85, 4434 (2000).

[12] W.D. Goldberger and M.B. Wise, Phys. Rev. D 60, 107505
(1999); Phys. Rev. Lett. 83, 4922 (1999).

[13] S.J. Huber and Q. Shafi, Phys. Rev. D 63, 045010 (2001).

[14] T. Gherghetta and A. Pomarol, Nucl. Phys. B602, 3 (2001).

[15] G.R. Dvali and A. Vilenkin, Phys. Rev. D 64, 063509 (2001).

[16] S. Coleman, Phys. Rev. D 15, 2929 (1977); S. Coleman and C. Callan, ibid. 16, 1762 (1977). 